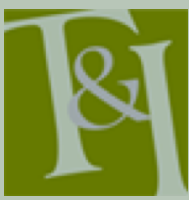

The International Journal for Translation \& Interpreting Research trans-int.org

\title{
Translating the Hopkins Symptom Checklist-25 (HSCL-25) into Dinka, a South Sudanese tribal language
}

\author{
Martha B. Baird \\ University of Kansas Medical Center \\ mbaird@kumc.edu
}

\author{
Elizabeth Skariah \\ CVS Minute Clinic, USA \\ eskariah@,att.net
}

DOI: 10.12807/ti.108202.2016.a07

\begin{abstract}
Translation and cultural adaptation of screening tools is an essential first step when assessing the mental health status of those from diverse cultures. This article presents a translated and culturally adapted version of the Hopkins Symptom Checklist-25 (HSCL-25), a measure of anxiety and depression, from English into Dinka, a South Sudanese tribal language. The HSCL-25 was translated and blindly back translated independently by two native South Sudanese from the Dinka tribe, a man and a woman, who came to the US as refugees. Once consensus was reached between the two translators, the Dinka translation was reviewed by a focus group of five native Dinka speakers. The original English version and the newly translated Dinka version were critically evaluated for cultural relevance and semantic congruence. Adjustments were made as a result of the focus group discussion, and a final Dinka version was created.
\end{abstract}

Keywords: Sudanese refugees, Dinka, depression, anxiety, Hopkins Symptom Checklist 25, HSCL-25, translation of mental health screening instruments

\section{Introduction}

It is essential that mental health screening instruments be evaluated and adapted for linguistic and conceptual equivalence for use with populations from other cultures. "Culture and language are intimately interdependent" (Schrauf, 2000, p. 389); therefore accurate cultural and linguistic translation is essential to assure meaningful results. Psychological constructs are not universal across cultures. Research in transcultural psychiatry has revealed that psychopathology varies across cultures, yet most screening instruments have been "developed by Western professionals using Western concepts and scale formats, based on Western norms and expectations" (Marsella \& Kameoka, 1989, p. 237). Unless mental health assessment instruments are adapted for ethnocultural variation, the validity and reliability of the conclusions are questionable (Marsella \& Kameoka, 1989).

This study presents the process of translating and culturally adapting the English version of the Hopkins Symptom Checklist-25 (HSCL-25) into Dinka, a South Sudanese tribal language. The ultimate goal is to create a 
linguistically valid and culturally relevant version of the HSCL-25 for use in clinical settings and research studies with South Sudanese Dinka speakers.

\subsection{Background}

The prolonged Sudanese civil wars have led to the death and displacement of millions (Al-Tohami, 2011). Sudanese refugees are scattered all over the globe. The Dinka, one of the largest tribes from South Sudan, have endured years of persecution by the northern Arab Sudanese militia for their Christian beliefs and practices and for access to their oil-rich and fertile lands (Peterson, 1999).

The original conflict in the Sudan began when colonization by the British in the south and Egypt in the north ended in 1956. There has been an almost constant state of civil war since. South Sudan officially seceded from the north in 2011 and became an independent nation (Gettleman, 2011). However, shortly after independence, war broke out again in December 2013 between the two ruling tribes, the Dinka and the Nuer, leaving thousands more displaced and traumatized (Kushkush, 2013).

Refugees experience a heightened risk of mental health conditions related to the trauma of forced migration. The World Health Organization (WHO) reports that more than $50 \%$ of refugees present with mental health problems ranging from chronic mental disorders to trauma and distress (World Health Organization (WHO), 2012). Depressive disorders among refugees range from $42 \%$ to $89 \%$, and rates of post-traumatic stress disorder (PTSD) are estimated to be $50 \%$ or higher (Jaranson et al., 2004; Silove, Sinnerbrink, Field, Manicavasager, \& Steel, 1997). In addition, the mental health of refugees shows a downward trend the longer they are in the host country (Barnes, Harrison, \& Heneghan, 2004; Portes, Fernandez-Kelly, \& Haller, 2009). Thousands of Sudanese refugees have been relocated to Western countries such as the United States, Australia, and the United Kingdom for permanent resettlement. Therefore, it is imperative that mental health instruments used to screen refugees be accurately translated and culturally relevant.

A further barrier to providing mental health assessments among these refugees is the fact that although Arabic is the official language of Sudan, there are estimated to be over 400 different tribes in the country, each with its own language (Peterson, 1999). The Dinka tribe is one of the largest, and their primary language is also called Dinka (Deng, 1984). The Dinka language was first written by German and Italian scholars and missionaries between 1928 and 1950 for purposes of translating the Christian Bible (Idris, 2004). Consequently, it is both an emerging language and one with limited development. After the South gained independence from the North, English was chosen as the second unifying language for South Sudan. However, many South Sudanese, and especially those from rural areas, have little or no formal education and are illiterate and unable to read or write any language.

The trauma that South Sudanese refugees have gone through puts them at risk for anxiety, depression, and PTSD (Schweitzer, Melville, Steel, \& Lacherez, 2006; Tempany, 2009). Translating instruments into the native language is a necessary first step before using them to measure symptoms of anxiety and depression among these refugees. Since many South Sudanese refugees either do not speak English or are unable to read or write their native language, an interpreter is often utilized, especially for medical matters.

Several authors have examined the communication patterns between refugees, mental health providers, and interpreters and found interpreters can exert a strong influence in this interaction (Bot, 2005, Mirdal, Ryding, \& Sondej, 2012). Interpreters often have their own style of interpretation and may include their own perspectives into the interaction. Bot (2005) found that 
"interpreters frequently stray from direct translation style and often interject their own feelings, opinions, memories, and preconceptions" (p. 243). This could inadvertently create response bias in the interviewee, which could lead to invalid responses. Thus it follows that the more clearly a written instrument is translated into the native tongue, the less scope exists for interpreter discretion in the interview process, thereby minimising communication error.

It is imperative to determine consistency and equivalency between the original language version and the translated version of the instrument in order to address the mental health needs of displaced South Sudanese. The Hopkins Symptom Checklist (HSCL) was chosen as a measure for anxiety and depression in South Sudanese refugees. This instrument has been translated and found valid and reliable in a number of refugee populations.

\subsection{Literature Review}

In a selective review of studies concerned with the mental health of Sudanese refugees, Tempany (2009) found high rates of psychopathology, particularly PTSD and depression. Yet the refugees maintained the ability to function and were more concerned with current stressors within the family than with past trauma. The authors caution that serious threats to validity may occur when using quantitative measures with refugee populations, such as the Sudanese, because language and conceptual meanings are so different. In addition, the fact that most Sudanese are unable to use paper and pencil surveys due to illiteracy means that an interpreter is required to communicate very complex constructs associated with mental health and illness.

In a study of the psychometric properties of the HSCL-37A for refugee adolescents in Europe, the authors found the most frequently reported symptoms were somatic complaints, anxiety, depression, and post-traumatic stress reactions. Unaccompanied refugee minors were found to have an especially high risk for developing psychopathology due to separation from primary caregivers, exposure to sequential stressful events, limited educational opportunities, and conditions in asylum centres during a very vulnerable developmental period (Bean, Derluyn, Eurelings-Bontekoe, Broekaert, \& Spinhoven, 2007).

In a study of 63 Sudanese refugees who relocated to Australia, structured interviews were conducted using bilingual interpreters in Arabic and English in order to determine various demographic characteristics, pre-migration trauma, level of social support, and post-migration adaptation and difficulties, which were measured by the HSCL-37, Harvard Trauma Questionnaire (HTQ), and the Post-Migration Living Difficulties Checklist (Schweitzer et al., 2006). Several factors were found to be associated with depression and anxiety in this group. These included being single, experiencing trauma prior to relocating, being female, and the length of time the refugee had been resettled. Also of note, the women in this study exhibited more symptoms of PTSD, depression, and anxiety than did the men.

In another study of the mental health needs of South Sudanese living in post-conflict South Sudan, the HTQ was used to measure PTSD, and the Arabic and Bari language version of the HSCL-25 was used to measure depression. In this study the authors found that over one-third of respondents met the criteria for PTSD, and 50\% met criteria for depression (Roberts, Damundu, Lomoro, \& Sondorp, 2009).

Fox and Willis (2009) conducted a mental health assessment of Sudanese refugees in the US preparing to have dental restorations. The authors noted that no routine mental health screenings or services were offered to refugees resettled to the US. They sought to determine a "baseline level of reported psychiatric distress" in these participants prior to completing any dental work. 
The researchers interviewed 33 potential participants using the HTQ and the English version of the HSCL-25 as part of the pre-dental screening. Participants included 15 men from the Dinka tribe and 18 men and women from the Nuer tribe. None of the women and few of the men in this study were able to read in either their native language or in English. The three male interpreters were also unable to read their native languages. Therefore, the English versions of the self-report instruments were administered verbally.

The results of this study showed a rate of PTSD at 3\%, depression at $12 \%$, and anxiety at $9 \%$ for this sample (Fox \& Willis, 2009). The researchers reasoned that the scores for these disorders were low for several reasons. These included small sample size, the possibility that these psychiatric disorders were demonstrated more through somatic rather than emotional symptoms, and the potential incongruence of the original meaning of the oral interpretations of the English instruments. The authors concluded that, "mental health practitioners must consider individual clinical presentation within the context of the refugees' cultural schemas, cultural idioms of distress, and overall cultural meaning system" (p. 175). They also stressed the importance of quality instruments for mental health screening of refugees from diverse cultures.

Most studies about the mental health of Sudanese refugees indicate varying levels of anxiety and depression. The HSCL-25 seems to be a popular assessment tool in the investigation of refugee mental health; however, very few studies used a translated and culturally adapted version, even though several mentioned the importance of this in order to adequately capture the cultural and social nuances associated with constructs of mental distress. This is the first study we are aware of in which the HSCL-25 has been culturally adapted and translated into the Dinka language.

\section{The Hopkins Symptom Checklist (HSCL) and its translations}

The HSCL was originally developed at Johns Hopkins University in 1954 for use in primary care settings. There are several versions of the HSCL that include from 25 to 90 items. The most commonly used 90-item checklist, the SCL-90, was developed by Derogatis, Lipman, and Covi in 1971 (Derogatis, Lipman, Richels, Ulenhuth, \& Covi, 1974), and includes measures of psychotic and paranoid symptoms in addition to those of anxiety and depression. The HSCL-25 has been found useful for use in medical settings to identify patients with emotional distress (Hesbacher, Rickels, Morris, Newman, \& Rosenfeld, 1980; Mollica, McDonald, Massagli, \& Silove, 2004). Both the HSCL-25 and the HSCL-37 have been used to assess mental health indicators in various populations. Both versions measure indicators of anxiety and depression. The HSCL-37 also measures 12 factors associated with somatization.

The HSCL-25 consists of two parts: Part I has 10 items for anxiety symptoms; Part II has 15 items for depression symptoms. The scale for each question includes four response categories ("Not at all", "A little", "Quite a bit", and "Extremely", rated 1 to 4, respectively). The HSCL-25 has consistently shown, in diverse populations, that the total score (Parts I and II) is highly correlated with severe emotional distress of unspecified diagnosis, and the depression score (Part II) is correlated with major depression as defined by the Diagnostic and Statistical Manual (DSM-IV-R) of the American Psychiatric Association (American Psychiatric Association (APA), 2000). In addition, the HSCL-25 is sensitive to symptom change over time, 
providing quantitative estimates of improvement with intervention (Mollica et al., 2004).

Translated into over 30 languages (Lavelle, 2011), the HSCL has been found to be reliable and valid in a variety of cultural groups including traumatized refugee populations (Kleijn, Hovens, \& Rodenburg, 2001; Mollica et al., 2004). In one of the first adaptations for use with refugees, (Mollica, Wyshak, de Marneffe, Khuon, \& Lavelle, 1987), the HSCL-25 was translated and validated with three groups of Indochinese refugees (Cambodian, Laotian, and Vietnamese). The authors found that the instrument was simple and reliable and was an effective screening method for anxiety and depression in these traumatized populations.

In a review of 47 translation studies, Maneesriwongul and Dixon (2004) found diverse methods of varying quality for translating instruments into target languages. They concluded that information about the translation process was inadequate and recommended that all studies involving instrument translation should include information about the methods used to translate, even when the study used an instrument previously translated by other researchers, especially when this information had not been previously published in an accessible source.

A study was conducted to test the psychometric properties of the Hopkins Symptom CheckIist-37 (HSCL-37A) by comparing versions in three different languages in a group of refugee adolescents, immigrants, and natives in Holland and Belgium (Bean et al., 2007). Adolescents from 35 different countries took the French version; those from 50 different countries took the English version; and another group from 20 different countries took the Arabic version. They identified that the HCSL-37A is a reliable and valid instrument to use with culturally diverse adolescents despite the diversity in translations. However, they cited a lack of back-translation of the French and Arabic versions as a potential limitation of the study.

In a study by Hoffmann et al. (2006), the HSCL-25 was translated into Russian by two native Russian speakers. The two goals of this study were to keep the translation at a sixth grade reading level and to maintain semantic equivalence. A third bilingual individual translated the newly translated version back into English. The Russian version was then modified after a review of the English back-translation. Finally, a new Russian version was tested on a convenience sample of ten Russian-speaking adults, which helped the researchers clarify several of the items on the instrument, which resulted in the final product. The data were statistically analyzed, and it was determined that the instrument had internal consistency and that the psychiatric constructs were preserved in the new Russian version. The conclusion was that a lower percentage of Russian individuals were diagnosed with anxiety and depression using this scale than the researchers had predicted.

In another study involving translation and validation of the HSCL-25 from English into Tibetan (Lhewa, Banu, Rosenfeld, \& Keller, 2007), one bilingual individual translated the original English version of the HSCL-25 into Tibetan, and another experienced translator back-translated the Tibetan version into English. Next, the Tibetan version was tested on a sample of 57 Tibetan refugee participants, and the participants were also evaluated by a psychiatrist fluent in Tibetan who was unaware of the participants' scores on the HSCL-25. The researchers then evaluated the data on SPSS for Windows, version 12.0, establishing internal consistency using Cronbach's alpha. The HSCL-25 also showed high rates of sensitivity and specificity when compared with the independent clinical diagnosis of the psychiatrist.

The 25-item version of the HSCL was chosen for translation and cultural adaptation by the authors for use in a mental health intervention with South 
Sudanese refugee women. In this pilot study the Dinka version of the HSCL25 was administered to the women pre, and post-intervention and the scores were compared to determine if the intervention had a significant effect on the women's symptoms of anxiety and depression. The results of this study have been submitted for publication (Baird, et al., forthcoming).

\subsection{Challenges Associated with Instrument Translation}

According to Polit and Beck (2012), translating an instrument for use in a different language and culture is often as much work as creating the original instrument. This is because much care needs to be taken to communicate concepts clearly, with the same meaning as in the original language, and to ensure that concepts in one language and culture apply to the language and culture for which the instrument will be used (Flaherty et al., 1988). In order for a translated instrument such as the HSCL-25 to have cross-cultural validity, it is necessary to establish both conceptual and semantic equivalence.

Conceptual equivalence refers to how well an instrument measures the same construct in the original culture and language and the target culture and language (Flaherty et al., 1988). In the case of the HSCL-25, reaching conceptual equivalence between the English and Dinka versions would mean that depression and anxiety could be detected equally by both versions. This seems straightforward until one considers that the way those from Englishspeaking countries experience or describe anxiety or depression may be different from the way individuals from the African Dinka culture would describe or understand these constructs. Determining if constructs in the original instrument have the same meaning in the target language and culture is a preliminary step in the translation process.

Semantic equivalence means that what is translated has the same meaning as the words used in the instrument in the original language (Flaherty et al., 1988). Polit and Beck (2012) stress the importance of keeping the meaning the same rather than creating a literal translation. Therefore, translating an instrument word-for-word is not recommended because the questions or items may not be communicated correctly, especially if any idioms were used in the source language.

Jones, Lee, Phillips, Zhang, and Jaceido (2001) describe a sequence of translation and back-translation to achieve semantic equivalence. First, the instrument is translated from the source language to the target language. Next, the instrument is back-translated - i.e. translated from the target language into the source language - by a translator or translators who do not know the original wording of the instrument. If the back-translation is a very close match to the original wording, it is a good indicator of semantic equivalence. Other important considerations include having a minimum of two bilingual and bicultural translators, preferably individuals who are also trained as professional translators. The individual who is developing the instrument should discuss it with each translator, making sure that the translators understand the intended purpose of the instrument, any items that include idioms and colloquialisms, and the intended reading level of the instrument.

After the back-translation of the instrument has been compared with the original version, a committee consisting of at least three bilingual individuals (not including the original translators) should review the translated version of the instrument. This committee should be given full access to the original instrument, the translated instrument, and the back-translation, and the committee should be given adequate time to discuss the instruments (Polit \& Beck, 2012). 


\section{Cross-cultural Translation and Adaptation of the HSCL-25 from English into Dinka}

The design of this study entailed a cross-cultural instrument translation of the HSCL-25 (English to Dinka), a corresponding back-translation (Dinka to English), and subsequent validation of the translated Dinka version of the instrument with a focus group from the target population. The goal of this translation research project was to achieve equivalence between the original version and the translated version of the scale (Streiner \& Norman, 2005). The Harvard Program in Refugee Trauma Manual, Measuring Torture, Measuring Trauma, was used as a guide for this translational study (Mollica et al., 2004).

The HSCL-25 was translated using a method endorsed by the World Health Organization (WHO) to establish cultural adaptation of an instrument. This method includes five steps: (1) forward translation; (2) expert panel; (3) back-translation; (4) pre-testing and cognitive interviewing with the target population, and; (5) development of a final version (World Health Organization, 2014).

Two bilingual and bicultural experts, a man and woman who came to the US as South Sudanese refugees, were hired as independent translators for this research project. Both of these translators had worked with the first author previously on translation and research projects (Baird, 2011; Baird \& Boyle, 2012; Baird et al., 2015). The male translator, one of the few South Sudanese refugees in the local area who was able to write the Dinka language, translated the English version into written Dinka. He had learned to write Dinka in the Kakuma refugee camp in Kenya before coming to the US as one of the socalled 'Lost Boys of Sudan'. The woman, a professional certified translator and interpreter with a degree in nursing from a Sudanese university, independently back-translated the Dinka version into English without viewing the original English version. The translators were directed not to discuss the translation or back-translation with each other during this process. The first author was available to both translators to answer any questions during the translation process.

After the two translators had completed their respective tasks, the authors met with them to compare and review the resulting versions - Dinka and reverse-English - of the HSCL-25. The results were discussed, discrepancies between the two texts were noted, and consensus was reached on a semantic translation of the instrument. The second author acted as an observer during this meeting, taking notes of any discrepancies in discussion of the translation. After agreement was reached between the two translated versions, a final translated Dinka version of the HSCL-25 was created.

The next step was to present this proposed translation to a group of native Dinka speakers. The authors and the female translator conducted a 90-minute focus group with five South Sudanese adult refugees (two men and three women) whose primary language was Dinka, to gain their perspectives on the linguistic and semantic equivalence of the translated Dinka version of the HSCL-25.

The audio-recorded focus group was held in a private room at the Sudanese Community Church after the Sunday worship service. The University Institutional Review Board (IRB) approval was obtained and approved recruitment and consent procedures. Since most South Sudanese refugees are illiterate, informed consent was verbally obtained by consensus. At the beginning of the focus group, a statement was read aloud explaining the purpose of the study and the participant's role in the focus group, emphasizing that the purpose was to clarify the meanings of the survey items, not to answer the survey questions with personal responses. No personal identifiers were 
used that linked the participants with any of their data, and participants were informed that they could withdraw from the study at any time. Each focus group participant was offered a $\$ 25$ gift card as an incentive for participation. One participant declined the incentive for unknown reasons.

The authors each listened to the audio recording and met together and discussed the session. Further adjustments were made to the instrument as necessary based on the input of the participants. The results of these discussions with the two translators and the focus group participants are presented in Appendix A.

After the focus group session, the authors met with both translators again to make adjustments to the Dinka version of the HSCL-25 based on input from the focus group participants. It was necessary to purchase a software program, Keyman Desktop Professional 8.0 (Tavultesoft, 2012), which allowed the author to type the Dinka version because many of the characters were not available on the US keyboard.

\section{Findings}

During the course of this study, there were a total of six meetings to compare and reconcile the translations before the final new Dinka version of the HSCL25 was completed. The first meeting occurred with both translators to reach agreement between the forward and back-translated versions. The second meeting was with the focus group, and four more meetings were held with the two translators to complete the final typed Dinka version.

The HSCL-25 is divided into two subscales: the first is labelled Anxiety Symptoms, and the second is Depression Symptoms. The translators informed us that there is no word in the Dinka language for anxiety or depression. It was difficult to translate something for which there is no comparative word and concerns a condition of which many people from this cultural group are not aware. Because of this, the constructs of anxiety and depression had to be described to the group. The focus group participants decided on the phrase "Tak aretic", which most closely represented the construct of depression to Dinka speakers; however the construct of anxiety was unable to be translated.

Symptoms concerned with physical changes were relatively straightforward to translate, e.g. "headaches", "poor appetite", or "difficulty falling asleep". However, items that had a more subjective or emotional nature proved more difficult. For instance, there were several items on the anxiety subscale that were difficult to translate from English into Dinka. Items (4) "Nervousness or shakiness inside", (6) "Trembling", and (9) "Spell of terror or panic", took quite a bit of explanation since there is no lexical equivalent in the Dinka language. We had to break down the terms and give examples in order to explain these words or constructs. It was also discovered that the syntax, in the Dinka language is very different than in English.

On the depression subscale, two of the items were especially difficult to translate and reach conceptual equivalence. The translators shared with us that they were uncomfortable discussing item 14, "Loss of sexual interest or pleasure", in mixed-gender company. They shared that it is not socially acceptable to discuss sex or topics related to sexual matters in the presence of the opposite gender, or even for older women to discuss sex with younger women.

Item (18), "Feeling blue", had to be explained to the translators and focus group participants. This English idiom would likely be meaningless to Dinka speakers. We explained that the term "feeling blue" is an English language metaphor that corresponds to an emotional, subjective experience associating 
a mood with a colour. The Dinka translation was changed to mean "Feel in sorrow; sympathy".

During the course of this study we learned from the two translators and focus group participants that there are three main dialects of the Dinka language: Bor, Agar, and Bhar-Gazal. The focus group participants reported that the written word is usually the same in all three dialects, but the pronunciation of many words is different. In the focus group, four participants spoke the Bor dialect, and one spoke the Bhar-Gazal dialect. Hearing from individuals who spoke different dialects was helpful as we worked to achieve the best translation for multiple dialects, although mainly focusing on the Bor dialect.

Both of the translators and all of the focus group participants reported that the English language has many more descriptive words, especially those pertaining to symptoms and other medical terminology, than does the Dinka language. Another challenge was that many of the characters are quite different in Dinka than in the English language. Thus, we had to type our final text using the Dinka version of the Keyman Desktop Professional 8.0 software program (Tavultesoft, 2012)

A table containing the original English version of the HSCL-25, the Dinka translation, the English back-translation, and other discussions that took place, is presented below.

Table 1. Hopkins Symptom Checklist-25 (HSCL-25) translation discussions.

\begin{tabular}{|ll|}
\hline \multicolumn{1}{|c|}{$\begin{array}{c}\text { HSCL-25 Original English } \\
\text { and } \\
\text { Dinka translation }\end{array}$} & \multicolumn{1}{c|}{$\begin{array}{c}\text { Dinka translation (in quotes) } \\
\text { and }\end{array}$} \\
& Pocus group discussion
\end{tabular}




\begin{tabular}{|c|c|}
\hline $\begin{array}{l}\text { Feeling restless or can't sit still. } \\
\text { Уغn ci nyuc ye leu. }\end{array}$ & $\begin{array}{l}\text { "Are you able to sit down by yourself?" Initially this was } \\
\text { translated into "loss of interest", "hyper". Much } \\
\text { discussion. Focus group decided that this is the most } \\
\text { polite way to translate this item because only children } \\
\text { are thought to be hyper. Consensus reached. }\end{array}$ \\
\hline \multicolumn{2}{|c|}{ Part II - Depression Symptoms } \\
\hline $\begin{array}{l}\text { Depression } \\
\text { Tak aretic. }\end{array}$ & $\begin{array}{l}\text { "Sad thoughts". Much discussion about what depression } \\
\text { is and how it is a construct. The depression symptoms } \\
\text { were much more easily translated than were the anxiety } \\
\text { symptoms. }\end{array}$ \\
\hline $\begin{array}{l}\text { Feeling low in energy, slowed } \\
\text { down. } \\
\text { Bi yin luany gusp. }\end{array}$ & $\begin{array}{l}\text { "No power, weakness, not enough energy". Much } \\
\text { discussion of this one before consensus was reached. }\end{array}$ \\
\hline $\begin{array}{l}\text { Blaming yourself for things. } \\
\text { Ba röt gok abac. }\end{array}$ & $\begin{array}{l}\text { "Blame yourself; feel guilty". Little discussion. } \\
\text { Consensus reached quickly. }\end{array}$ \\
\hline $\begin{array}{l}\text { Crying easily. } \\
\text { Ba däc dhiäau. }\end{array}$ & $\begin{array}{l}\text { "Easy to cry". Little discussion. Consensus reached } \\
\text { quickly. }\end{array}$ \\
\hline $\begin{array}{l}\text { Loss of sexual interest or pleasure. } \\
\text { Cen thiäk e kam tik kene moc. }\end{array}$ & $\begin{array}{l}\text { "Feel close to your spouse" or "Be in the room [with } \\
\text { another person]". In this culture, sex is not directly } \\
\text { discussed. }\end{array}$ \\
\hline $\begin{array}{l}\text { Poor appetite. } \\
\text { Ba piöu cen cäm. }\end{array}$ & $\begin{array}{l}\text { "Poor appetite". Little discussion. Consensus reached } \\
\text { quickly. }\end{array}$ \\
\hline $\begin{array}{l}\text { Difficulty falling asleep, staying } \\
\text { asleep. } \\
\text { Bä nyin cen nin }\end{array}$ & $\begin{array}{l}\text { "Insomnia". Little discussion. Consensus reached } \\
\text { quickly. }\end{array}$ \\
\hline $\begin{array}{l}\text { Feeling hopeless about the future. } \\
\text { Bä cen__ath. }\end{array}$ & $\begin{array}{l}\text { "Hopelessness, loss of hope". Little discussion. } \\
\text { Consensus reached quickly. }\end{array}$ \\
\hline $\begin{array}{l}\text { Feeling blue. } \\
\text { Dhien de piöu. }\end{array}$ & $\begin{array}{l}\text { "Feel in sorrow. Sympathy". Much discussion, especially } \\
\text { how "feeling blue" is an idiom in the English language } \\
\text { and means "sadness". Consensus reached. }\end{array}$ \\
\hline $\begin{array}{l}\text { Feeling lonely. } \\
\text { Bä nyin kok. }\end{array}$ & $\begin{array}{l}\text { "Loneliness". Little discussion. Consensus reached } \\
\text { quickly. }\end{array}$ \\
\hline $\begin{array}{l}\text { Thought of ending your life. } \\
\text { Bä tak cen ye nak röt. }\end{array}$ & $\begin{array}{l}\text { "Feel suicidal". Little discussion. Consensus reached } \\
\text { quickly. }\end{array}$ \\
\hline $\begin{array}{l}\text { Feeling of being trapped or caught. } \\
\text { Ba rot yok cike yin cï duut piny. }\end{array}$ & $\begin{array}{l}\text { "Feel like you're confined". More discussion before } \\
\text { agreeing on this translation. The first translation had a } \\
\text { connotation of being locked up in jail. }\end{array}$ \\
\hline $\begin{array}{l}\text { Worry too much about things. } \\
\text { Ba dier arëtic ne kay kedhie. }\end{array}$ & $\begin{array}{l}\text { "You worry about everything". Little discussion. } \\
\text { Consensus reached quickly. }\end{array}$ \\
\hline $\begin{array}{l}\text { Feeling no interest in things. } \\
\text { Ba gusp Locut. }\end{array}$ & $\begin{array}{l}\text { "Don't feel like doing anything. Not lazy, just not feeling } \\
\text { like you can do anything. No motivation". Some } \\
\text { discussion before consensus was reached. }\end{array}$ \\
\hline $\begin{array}{l}\text { Feeling everything is an effort. } \\
\text { Bi ke rieec yin dom rendëk. }\end{array}$ & $\begin{array}{l}\text { "Everything makes you tired". Little discussion. } \\
\text { Consensus reached quickly. }\end{array}$ \\
\hline $\begin{array}{l}\text { Feeling of worthlessness. } \\
\text { Ba röt yok ke yin ye ran abac. }\end{array}$ & $\begin{array}{l}\text { "Low self esteem. No value to yourself". Little } \\
\text { discussion. Consensus reached quickly. }\end{array}$ \\
\hline
\end{tabular}

\footnotetext{
${ }^{1}$ There was considerable discussion about this item. The translators reported that "sex is not talked about". A couple would simply talk about being in their bedroom. The female translator stated, "Sex is not that important to us. We go long periods of time without sexual relations." The interpreter prefaced this question to the focus group so that they would not be offended or embarrassed. It is considered appropriate for a man to be sexually active, but not a woman. Women are not thought to have sexual desire. When a woman's daughter gets married, the sex life of the mother is considered to end and she stops having sex with her husband. Therefore, it would be inappropriate to ask this question of a woman. It is considered inappropriate for a woman and a man to discuss this topic together. For example, a female healthcare provider asking a male patient this question would be inappropriate.
} 


\section{Discussion}

Very few authors publish the process of instrument translation, and when they do the process varies considerably. There is a need for consensus among researchers as to how to achieve high quality instrument translation in crosscultural research (Maneesriwongul \& Dixon, 2004). In this translation study several items on both the anxiety and depression subscale were found to be either linguistically difficult or culturally inappropriate for use with this African population. Translation studies with other refugee populations have also found several items in the HSCL-25 to be linguistically and culturally inappropriate. Similar to our findings, in a translation and cultural adaptation of the HSCL-37 with a sample of Congolese adolescents, the authors recommended eliminating item (14), "Loss of sexual interest or pleasure", for use in this population (Mels, Derluyn, Broekaert, \& Rosseel, 2009). Another translation study with a Tibetan population also found item 14, to be culturally inappropriate as sexual activity is rarely discussed in Tibetan culture (Lhewa et al., 2007). In the Tibetan study even when the authors removed the item to avoid making respondents uncomfortable, they found the consistency of the subscale to be maintained despite the removal (Lhewa et al., 2007).

The primary author of this study recently completed a translation and cultural adaptation of the HSCL-25 with a Bhutanese refugee population and also found this item (14) to be socially inappropriate, especially in mixedgender company (Baird, LeMaster, \& Harding, 2016). We left this item in the HSCL-25 Dinka translation; however, in the future these items may need to be either rephrased or eliminated to avoid cultural inappropriateness.

Due to the literacy level of the Dinka, the instructions to the HSCL-25 were changed from self-report to reflect verbal administration of the instrument. This adaptation was also made for the Tibetan version (Lhewa et al., 2007) and should be considered in future translations for populations from underdeveloped countries to keep the instrument congruent with educational and reading levels.

\section{Conclusion}

In this study the HSCL-25 was translated into the Dinka language, and then back-translated into English. The two texts were compared to reach semantic consensus and a final Dinka version was proposed. A focus group of Dinka refugees reviewed the final Dinka version for translation accuracy and cultural appropriateness. As a result of the translation process and focus group feedback, it was concluded that item 14, "Loss of sexual interest or pleasure", be removed from the scale to make the instrument culturally relevant and acceptable. It is also recommended that the instructions for completing the instrument be changed from self-report to verbal administration due to the literacy level of this population.

An important next step will be to evaluate the new Dinka version of the HSCL-25 for reliability with a sample of individuals from the target population. Administering the instrument to a larger sample of individuals should be done to determine whether the instrument measures the intended constructs.

The need to demonstrate positive outcomes for therapeutic interventions is becoming more pressing in all mental health services, including those for refugees (Mollica et al., 2004). There are very few validated instruments for screening refugees for mental health conditions (Jacobsen \& Thoresen, 2010) and a critical lack of culturally-congruent mental health treatments. 
Standardized procedures such as the Translation Monitoring Form (van Ommeren et al., 1999) could enhance the reliability and validity of translation study results. This study presents the process involved in creating a linguistically and culturally relevant survey instrument for use with South Sudanese refugees.

\section{References}

Al-Tohami, K. (2011). Migration in Sudan: A country profile 2011. Khartoum: International Organization for Migration.

American Psychiatric Association (APA) (2000). Diagnostic and statistical manual of psychiatric disorders (4th-TR, ed.). Washington, DC: American Psychiatric Association (APA).

Baird, M. B. (2011). Lessons learned in working with translators and interpreters from the Dinka tribe of southern Sudan. Journal of Transcultural Nursing, 22(2), 116-121.

Baird, M. B., Bimali, M., Cott, A., Brimacombe, M., Petty, T.R., \& Daley, C. M. (forthcoming). Healthy Sudanese families: A mental health intervention for refugee women. (Manuscript submitted for publication).

Baird, M. B., \& Boyle, J. S. (2012). Well-Being in Dinka refugee women of Southern Sudan. Journal of Transcultural Nursing, 23(1), 14-21. doi: $10.1177 / 1043659611423833$

Baird, M. B., Domian, E. W., Mulcahy, E. R., Jemutai-Tanui, G., Mabior, R., \& Filippi, M. K. (2015). Creating a bridge of understanding between two worlds: Community-based collaborative-action research with Sudanese refugee women. Public Health Nursing. doi: 10.1111/phn.12172

Baird, M. B., LeMaster, J. W., \& Harding, A. (June, 2016). Translation and cultural adaptation of the Hopkins Symptom Checklist-25 (HSCL-25) into Nepalese for use with Bhutanese refugees. The Online Journal of Cultural Competence in Nursing and Healthcare (OJCCNH).

Barnes, D. M., Harrison, C., \& Heneghan, R. (2004). Health risks and promotion behaviors in refugee populations. Journal of Health Care for the Poor and Underserved, 15, 347-356.

Bean, T., Derluyn, I., Eurelings-Bontekoe, E., Broekaert, E., \& Spinhoven, P. (2007). Validation of the multiple language versions of the Hopkins Symptom Checklist-37 for refugee adolescents. Adolescence, 42(165), 51-71.

Deng, F. M. (1984). The Dinka of Sudan. Prospect Heights, Illinois: Waveland Press, Inc.

Derogatis, L. R., Lipman, R. S., Richels, K., Ulenhuth, E. H., \& Covi, L. (1974). The Hopkins Symptom Checklist (HSCL): A self-report inventory. Behavioural Sciences, 19, 1-15.

Flaherty, J., Gaviria, M., Pathak, D., Mitchell, T., Wintrob, R., Richman, J., \& Birz, S. (1988). Developing instruments for cross-cultural psychiatric research. The Journal of Nervous and Mental Disease, 176(5), 257-263.

Fox, S. H., \& Willis, M. S. (2009). Initiatory mental health assessments for Dinka and Nuer refugees from Sudan. Journal of Immigrant \& Refugee Studies, 7, 159179.

Gettleman, J. (2011, July ). Sudan movement's mission is secured: Statehood. The New York Times online. Retrieved from http://www.nytimes.com/2011/ 07/09/world/africa/09sudan.html?ref=opinion\&_r=1

Hesbacher, P. T., Rickels, K., Morris, R. J., Newman, H., \& Rosenfeld, H. (1980). Psychiatric illness in family practice. Journal of Clinical Psychiatry, 41(1), 6-10.

Hoffmann, C., McFarland, B., Kinzie, J., Bresler, L., Rakhlin, D., Wolf, S., \& Kovas, A. (2006). Psychological distress among recent Russian immigrants in the United States. International Journal of Social Psychiatry, 52(1), 29-40. doi: $10.1177 / 0020764006061252$

Idris, H. F. (2004). Modern developments in the Dinka language. Göteborg Africana Informal Series-No. 3. Department of Oriental and African Languages. 
Gothenburg University. Gothenburg, Sweden. Retrieved from http://www. kultur.gu.se/digitalAssets/1309/1309450_modern-developments-dinka.pdf

Jacobsen, M., \& Thoresen, S. (2010). The validity of screening for post-traumatic stress disorder and other mental health problems among asylum seekers from different countries. Journal of Refugee Studies, 24(1), 171-186.

Jaranson, J. M., Butcher, J., Halcon, L., Johnson, D. R., Robertson, C., Savik, K., . . . Spring, M. (2004). Somali and Oromo refugees: Correlates of torture and trauma history. American Journal of Public Health, 94(4), 591-612.

Jones, P. S., Lee, J. W., Phillips, L. R., Zhang, Z. E., \& Jaceido, K. B. (2001). An adaptation of Brislin's translation model for cross-cultural research. Nursing Research, 50(5), 300-304.

Kleijn, W. C., Hovens, J. E., \& Rodenburg, J. J. (2001). Postraumatic stress symptoms in refugees: Assessments with the Harvard Trauma Questionnaire and the Hopkins Symptom Checklist-25 in different languages. Psychological Reports, 88, 527-532.

Kushkush, I. (2013). Political strife in South Sudan sets off ethnic violence. The New York Times. Retrieved from http://www.nytimes.com/2013/12/22/world/ africa/political-strife-in-south-sudan-sets-off-ethnic-violence.html? $\mathrm{r}=0$

Lavelle, J. (2011). Measuring trauma, measuing torture. In R. F. Mollica (Ed.), Global mental health: Trauma and recovery (pp. 506-537). Boston: Harvard Program in Refugee Trauma.

Lhewa, D., Banu, S., Rosenfeld, B., \& Keller, A. (2007). Validation of a Tibetan translation of the Hopkins Symptom Checklist-25 and the Harvard Trauma Questionnaire. Assessment, 14(3), 223-230. doi: 10.1177/1073191106298876

Maneesriwongul, W., \& Dixon, J. K. (2004). Instrument translation process: A methods review. Methodological Issues in Nursing Research, 48(2), 175-186.

Marsella, A. J., \& Kameoka, V. A. (1989). Ethnocultural Issues in the Assessment of Psychopathology. Washington, D. C.: American Psychiatric Press.

Mels, C., Derluyn, I., Broekaert, E., \& Rosseel, Y. (2009). Community-based crosscultural adaptation of mental health measures in emergency settings: validating the IES-R and HSCL-37A in Eastern Democratic Republic of Congo. Social Psychiatric Epidemiology, 45, 899-910.

Mollica, R. F., Wyshak, G., de Marneffe, D., Khuon, F., \& Lavelle, J. (1987). Indochinese versions of the Hopkins Symptom Checklist-25: A screening instrument for the psychiatric care of refugees. American Journal of Psychiatry, 144(4), 497-500.

Mollica, R. F., McDonald, L. S., Massagli, M. P., \& Silove, D. M. (2004). Measuring trauma, measuring torture: Instructions and guidance on the utilization of the Harvard Program in Refugee Trauma's Version of the Hopkins Symptom Checklist-25 (HSCL-25) \& The Harvard Trauma Questionnaire (HTQ). Cambridge, MA: Harvard Program in Refugee Trauma.

Peterson, D. (1999). Inside Sudan: Political Islam, conflict, and catastrophe. Boulder: Westview Press.

Polit, B., \& Beck, C. (2012). Nursing research: Generating and assessing evidence for nursing practice (9th ed.). Philadelphia, PA: Lippincott Williams \& Wilkins.

Portes, A., Fernandez-Kelly, P., \& Haller, W. (2009). The adaptation of the immigrant second generation in America: A theoretical overview and recent evidence. Journal of Ethnic and Migration Studies, 35(7), 1077-1104.

Roberts, B., Damundu, E. Y., Lomoro, O., \& Sondorp, E. (2009). Post-conflict mental health needs: A cross-sectional survey of trauma, depression, and associated factors in Juba, Southern Sudan. BioMed Central, 9(7), 1-10. doi: $10.1186 / 1471-244 x-9-7$

Schrauf, R. (2000). Bilingual and autobiographical memory: Experimental studies and clinical cases. Culture and Psychology, 6(4), 563-586.

Schweitzer, R., Melville, F., Steel, Z., \& Lacherez, P. (2006). Trauma, post-migration living difficulties, and social support as predictors of psychological adjustment in resettled Sudanese refugees. Australian and New Zealand Journal of Psychiatry, 40, 179-187.

Silove, D., Sinnerbrink, I., Field, A., Manicavasager, V., \& Steel, Z. (1997). Anxiety, depression and PTSD in asylum-seekers: Associations with pre-migration 
trauma and post-migration stressors. British Journal of Psychiatry, 170, 351357.

Streiner, D. L., \& Norman, G. R. (2005). Health measurement scales a practical guide to their development and use (3rd ed.). New York: Oxford University Press.

Tavultesoft. (2012). Keyman Desktop 8. 8. Retrieved March 24, 2014, from http://www.tavultesoft.com/keyman

Tempany, M. (2009). What research tells us about the mental health and psychosocial well-being of Sudanese refugees: A literature review. Transcultural Psychiatry, 46(2), 300-315.

van Ommeren, M., Sharma, B., Thapa, S., Makaju, R., Prasain, D., Bhattara, R., \& de Jong, J. (1999). Preparing instruments for transcultural research: Use of the Translation Monitoring Form with Nepali-speaking Bhutanese refugees. Transcultural Psychiatry, 36, 285-301.

World Health Organization (WHO) (2012). Mental health of refugees, internally displaced persons and other populations affected by conflict. Retrieved January 25, 2012, from http://www.who.int/hac/techguidance/pht/ mental_ health_refugees/en

World Health Organization (WHO) (2014). Process of translation and adaptation of instruments. Retrieved from http://www.who.int/entity/substance_abuse/ research_tools/translation/en 